PROCEEDINGS OF THE

AMERICAN MATHEMATICAL SOCIETY

Volume 129, Number 11, Pages 3445-3446

S 0002-9939(01)06226-8

Article electronically published on May 30, 2001

\title{
VARIATIONALLY COMPLETE REPRESENTATIONS ARE POLAR
}

\author{
ANTONIO J. DI SCALA AND CARLOS OLMOS
}

(Communicated by Wolfgang Ziller)

\begin{abstract}
A recent result of C. Gorodski and G. Thorbergsson, involving classification, asserts that a variationally complete representation is polar. The aim of this paper is to give a conceptual and very short proof of this fact, which is the converse of a result of Conlon.
\end{abstract}

The concept of a variationally complete action was introduced by R. Bott [B] in 1956. Two years later, Bott and Samelson [BS] proved that $s$-representations (i.e. isotropy representation of semisimple symmetric spaces) are variationally complete. This class of representations contains examples of what L. Conlon [C] called polar representations, or more generally hyperpolar actions. He proved that a hyperpolar action of a compact Lie group on a complete Riemannian manifold is variationally complete. Polar representations were classified by J. Dadok [D] who proved that they are orbit equivalent to $s$-representations (see also $[\mathrm{EH}]$ ). Recently, C. Gorodski and G. Thorbergsson classified variationally complete representations of compact Lie groups [GT]. From this classification they obtained that a variationally complete representation is also orbit equivalent to an $s$-representation (from this they obtained, with different methods, Dadok's list). So, they obtained the following equivalent theorem, some of whose history can be found in [TT, p. 196].

Theorem ([GT]). A variationally complete orthogonal representation of a compact Lie group is polar.

The object of this short note is to give a direct and geometric proof of the above theorem.

Recall that a compact connected Lie subgroup $G$ of $S O(n)$ acts polarly on $\mathbb{R}^{n}$ if there exists an affine subspace which meets orthogonally all $G$-orbits. This is equivalent to the fact that the tangent space $T_{v}(G . v)$ of a principal orbit G.v contains the tangent spaces of all orbits through points in the normal space $\nu_{v}(G \cdot v)$. The $G$-action is called variationally complete if any $G$-transversal Jacobi field (i.e. produced by variations of transversal geodesics) that is tangent to orbits at two points is the restriction of some Killing field on $\mathbb{R}^{n}$ induced by the action. Recall that a geodesic $\gamma(t)$ in $\mathbb{R}^{n}$ is $G$-transversal if it is orthogonal to the $G$-orbit through

Received by the editors November 9, 2000 and, in revised form, December 6, 2000.

1991 Mathematics Subject Classification. Primary 53C40; Secondary 53C35.

Key words and phrases. Variationally complete, polar representations, s-representations.

This research was supported by Universidad Nacional de Córdoba, CONICET, CONICOR, Secyt-UNC, ANPCyT and CIEM. 
$\gamma(t)$ for every $t$ (or equivalently, for some $t_{0}$ since a Killing field projects constantly to any geodesic).

Proof. Let $G$ be a compact connected Lie subgroup of $S O(n)$ such that the $G$-action is variationally complete, and let $v \in \mathbb{R}^{n}$ be a principal vector. Let $\xi_{v}$ be a normal vector to $G . v$ at $v$ whose shape operator $A_{\xi_{v}}$ has all eigenvalues different from zero. (Such normal vectors define an open and dense subset of the normal space. This is because the determinant of the shape operator is a nonzero polynomial on the normal space at a given point $v$, since $A_{v}=-I d$.) Let $c(s)$ be a curve in $G . v$ with $c(0)=v$ and such that $w:=c^{\prime}(0) \neq 0$ is an eigenvector of $A_{\xi_{v}}$ with associated eigenvalue $\lambda$. Extend $\xi_{v}$ to a parallel normal field $\xi(s)$ to $G . v$ along $c(s)$. Let us consider the variation by $G$-transversal geodesics given by $\gamma_{s}(t)=c(s)+t \xi(s)$ and set $J(t)=\left.\frac{\partial}{\partial s}\right|_{s=0} \gamma_{s}(t)=(1-t \lambda) w$. Then $J(t)$ is a $(G$-transversal) Jacobi field along the geodesic $\gamma_{0}(t)=v+t \xi_{v}$ of $\mathbb{R}^{n}$. Observe that $J(0)=w \in T_{v}(G . v)$ and $J(1 / \lambda)=0 \in T_{\gamma_{0}(1 / \lambda)}\left(G \cdot \gamma_{0}(1 / \lambda)\right)$. By the variational completeness of the $G$-action, $J$ is the restriction to $\gamma_{0}$ of a Killing field induced by $G$. Thus, for all $t$, $J(t)=(1-t \lambda) w$, and so $w$, belongs to $T_{\gamma_{0}(t)}\left(G \cdot \gamma_{0}(t)\right)$. Since the eigenvectors of $A_{\xi_{v}}$ generate $T_{v}(G . v)$, we obtain that $T_{\gamma_{0}(t)}\left(G \cdot \gamma_{0}(t)\right)=T_{v}(G \cdot v)$ for $t$ small. This easily implies that $G$ acts polarly.

Remark. It is also true that a variationally complete action of a noncompact Lie subgroup $G$ of $I s o\left(\mathbb{R}^{n}\right)$ is also polar. In fact, from [Di] (see also $\mathrm{O}$ ) there always exists a principal orbit $G$.p with a normal vector $\xi_{p}$ whose shape operator $A_{\xi}$ is positive definite (otherwise all $G$-orbits are parallel and totally geodesic), and so invertible. Then, the same proof applies.

\section{REFERENCES}

[B] Bott, R., An application of the Morse theory to the topology of Lie groups, Bull. Soc. Math. France 84 (1956), 251-281. MR 19:291a

[BS] Bott, R. and Samelson, H., Applications of the theory of Morse to symmetric spaces, Amer. J. Math. 80 (1958), 964-1029. Correction in vol. 83 (1961), 207-208. MR 21:4430. MR 30:589

[C] Conlon, L., Variational completeness and K-transversal domains, J. Differential Geom. 5 (1971), 135-147. MR 45:4320

[D] Dadok, J., Polar coordinates induced by action of compact Lie groups, Trans. Amer. Math. Soc. 288 (1985), 125-137. MR 86k:22019

[Di] Di Scala, A.J., Minimal homogeneous submanifolds in euclidean spaces, to appear in Annals of Global Analysis and Geometry.

[EH] Eschenburg, J.-H. and Heintze, E., Polar representations and symmetric spaces, J. reine angew. Math. 507 (1999), 93-106. MR 2000f:53072

[GT] C. Gorodski and G. Thorbergsson, Representations of compact Lie Groups and the osculating spaces of their orbits, Preprint Mathematisches Institut der Universität zu Köln (September 2000).

[O] Olmos, C., Orbits of rank one and parallel mean curvature, Trans. Amer. Math. Soc. 347, No. 8 (1995), 2927-2939. MR 96a:53069

[TT] Terng, C. L. and Thorbergsson G., Taut Immersions into Complete Riemannian Manifolds, Tight and Taut Submanifolds, MSRI Publications 32 (1997), 181-228. MR 99m:53122

Facultad de Matemática, Astronomía y Física, Universidad Nacional de Córdoba, 5000 Córdoba, Argentina

E-mail address: discala@mate.uncor.edu

Department of Mathematics, Ciudad Universitaria, 5000 Córdoba, Argentina

E-mail address: olmos@mate.uncor.edu 